Published in final edited form as:

Pituitary. 2012 December ; 15(4): . doi:10.1007/s11102-011-0367-3.

\title{
Phospho-histone $\mathrm{H3}$ (pHH3) immuno-reactivity as a prognostic marker in non-functioning pituitary adenomas
}

\author{
Erica Hightower, \\ Endocrinology, University of Texas Medical School at Houston, Houston, TX, USA \\ Maria E. Cabanillas, \\ Division of Endocrine Neoplasia \& Hormonal Disorders, Univeristy of Texas M. D. Anderson \\ Cancer Center, Houston, TX, USA
}

Greg N. Fuller,

Pathology, University of Texas M. D. Anderson Cancer Center, Houston, TX, USA

Ian E. McCutcheon,

Neurosurgery, University of Texas M. D. Anderson Cancer Center, Houston, TX, USA

Kenneth R. Hess,

Biostatistics, University of Texas M. D. Anderson Cancer Center, Houston, TX, USA

Komal Shah,

Radiology, University of Texas M. D. Anderson Cancer Center, Houston, TX, USA

Steven G. Waguespack,

Division of Endocrine Neoplasia \& Hormonal Disorders, Univeristy of Texas M. D. Anderson

Cancer Center, Houston, TX, USA

Lynda J. Corley, and

Pathology, University of Texas M. D. Anderson Cancer Center, Houston, TX, USA

Jessica K. Devin

Division of Endocrine Neoplasia \& Hormonal Disorders, Univeristy of Texas M. D. Anderson

Cancer Center, Houston, TX, USA

Jessica K. Devin: Jessica.Devin@Vanderbilt.edu

\section{Abstract}

Nonfunctioning pituitary adenomas (NFPA) are typically benign neoplasms that can cause significant morbidity through local mass effects. MIB-1/Ki-67 and p53 immuno-reactivity are used to predict aggressive behavior but have known limitations. No marker to date is widely used to reliably predict tumor progression. Phospho-histone $\mathrm{H} 3$ (pHH3) is a protein phosphorylated during chromatin condensation in mitosis, and thus anti-pHH3 immunocyto-chemistry is able to assess mitotic activity. Study objectives were to determine the relationship among pHH3, MIB-1/

\footnotetext{
(C) Springer Science+Business Media, LLC 2011

Correspondence to: Jessica K. Devin, Jessica.Devin@Vanderbilt .edu.

Present Address:

J. K. Devin, Division of Diabetes, Endocrinology and Metabolism, Vanderbilt University School of Medicine, 1215 21st Ave South, Suite 8017, Nashville, TN 37232, USA

Erica Hightower and Maria E. Cabanillas contributed equally to this manuscript.

Conflict of interest The authors declare they have no conflict of interest.

Ethical standards The research contained within this submission complies with the current laws set forth by the MDACC Institutional Review Board.
} 
Ki-67, and p53 in NFPA, and to evaluate the relationship between these indices and time to progression (TTP). Seventy-six patients with NFPA operated on by a single neuro-surgeon at University of Texas M. D. Anderson Cancer Center from 1992 to 2006 were identified from a database and met all criteria for inclusion in this clinicopathology study. PHH3, MIB-1/Ki-67, and p53 immuno-reactivity was evaluated in each case. Retrospective review was used to determine TTP. With 282 person-years of follow-up, 19 progression events were observed. A correlation was found between MIB-1/Ki-67 and p53 immuno-reactivity $(r=0.25, p=0.031)$. PHH3 did not correlate with either. When markers were dichotomized at the median, only MIB-1/Ki-67 correlated with TPP $(\log \operatorname{rank} p=0.018)$. Rank correlation analysis confirmed a significant inverse correlation between both MIB-1/Ki-67 (Dxy $=-0.33, p=0.036)$ and p53 (Dxy $=-0.40$, $0.016)$ immuno-reactivity and TTP. Our results support previous data suggesting that MIB-1/ $\mathrm{Ki}-67$ and p53 have clinical utility as prognostic markers for tumor progression. PHH3 did not prove to be associated with TTP in this retrospective study limited by few progression events.

\section{Keywords}

PHH3; Pituitary adenoma; MIB-1/Ki-67; p53

\section{Introduction}

Pituitary tumors account for nearly $10 \%$ of symptomatic intracranial tumors [1]. The majority of these tumors are histologically benign; however, they can be capable of aggressive growth and local destruction to nearby structures. Cell proliferation is one of the most important factors determining the behavior of many neoplasms [1]. Morphological criteria of nuclear pleomorphism, increased mitoses, and high cellularity characterizes a high proliferation rate [2]. The use of immuno-histochemical markers in determining prognosis has been studied in pituitary tumors with varying success. Previously investigated proliferative markers include MIB-1/Ki-67, the mitotic index, and p53 [3-5]. The 2004 WHO classification of pituitary adenomas includes an "atypical" variant, which behaves as an aggressive, invasive macroadenoma. Histologically, these tumors are defined by an elevated mitotic index, MIB-1/Ki-67 labeling index(LI) $>3 \%$, and extensive nuclear reactivity for $\mathrm{p} 53[3,5,6]$. Tumors with this pathological distinction may necessitate closer monitoring and more aggressive adjuvant therapy.

As of yet, there is not a commonly utilized marker that helps determine the likelihood of disease progression. This is particularly challenging in the setting of non-functioning pituitary adenomas (NFPA), as there is no hormone upon which to base a post-surgical cure. The most recent WHO classification lacks significant longitudinal follow-up. Furthermore, the presently available histologically markers are fraught with inconsistencies and previous studies have demonstrated variable results on their usefulness [7-9].

Phospho-histone $\mathrm{H} 3$ (pHH3), a protein involved in chromatin structure, is phosphorylated during chromatin condensation in mitosis. An antibody to $\mathrm{pHH} 3$ therefore has the potential to be a reliable method for identifying mitotic activity. PHH3 has proven to be an independent predictor of survival in patients with low and intermediate-grade infiltrating astrocytomas, and pHH3 has assisted in the grading of meningiomas [10, 11]. PHH3 immunostaining has proven to be particularly useful in identifying mitotic figures in tumors with dense cellularity, limited mitotic activity, and/or abundant apoptotic, pyknotic or distorted nuclei [11]. PHH3 has not previously been evaluated in the context of pituitary adenomas. This study was designed to determine the relationship of the pHH3 index to previous proliferative indices, and to evaluate the association of $\mathrm{pHH} 3$ immuno-reactivity with disease progression in NFPA. 


\section{Materials and methods}

\section{Case selection}

Cases with a diagnosis of pituitary adenoma dating from January 1, 1992 through December 31, 2006 were retrospectively identified from database review. All pathologic specimens were obtained following the initial transsphenoidal resection at University of Texas M. D. Anderson Cancer Center (MDACC) by a single neurosurgeon (IM), including specimens from initial surgery for de novo disease, as well as from subsequent surgery for residual or recurrent disease following previous resection at an outside institution. Only cases with sufficient material for immuno-histochemical examination were included.

Patients were excluded if they had a history of cranial radiation therapy or if they previously received medical therapy for their tumor. Also excluded were hormonally active tumors, including tumors which secreted ACTH, prolactin, growth hormone and/or TSH and resulted in the respective clinical syndromes of hormone excess; silent subtypes (positive hormonal staining without clinical evidence of hormone oversecretion) were included after the appropriate chart review.

The date of radiologic disease progression following initial surgery at MDACC was noted. This was defined as evidence of one of the following: a 30\% increase in tumor volume, a $10 \%$ increase in any diameter, or any detectable disease following a complete resection at MDACC. Patients must have had at least 1 year of post-operative follow up with MRI to allow this determination. The final number of cases demonstrating disease progression following initial surgery at MDACC was confirmed by independent radiology review. Additionally, a patient case was considered a progression event if the patient was evaluated at MDACC following resection(s) at an outside institution and necessitated further surgical resection at MDACC based upon the available radiologic imaging and clinical evaluation.

\section{Histological analyses}

Quantitation-Quantitation of all markers (PHH3, MIB-1/Ki-67, p53) was performed at $20 \times$ using a custom nuclear algorithm produced by the Genetix Ariol image analysis system (Applied Imaging; San Jose, CA).

PHH3 - Sections were stained for pHH3 with polyclonal antibody (Upstate Biotechnology; Waltham, MA) as previously described [10]. Sections were deparaffinized and rehydrated in graded alcohols. Antigen retrieval was performed by microwaving in $10 \mathrm{mM}$ sodium citrate, $\mathrm{pH} 8.0$ using a microwave pressure cooker. After incubation in 3\% hydrogen peroxide, sections were incubated overnight at 4 degrees Celsius in primary antibody at a 1:10,000 dilution. Staining was visualized using a kit from Dako (Carpinteria, CA). If no mitoses were identified, the sample was stained with an increased antibody concentration $(1: 2,000)$, in order to verify absence of mitoses as previously described [10].

All slides were viewed microscopically by a pathologist and areas with positive pHH3 mitotic figure staining were identified. In areas where mitotic figures were identified, ten $20 \times$ fields were quantitated, yielding total mitoses per 10 contiguous fields. The highest mitotic count found was used in the data analysis. PHH3 labeled mitoses stained with DAB were quantitated at $20 \times$ using a custom nuclear algorithm produced by the Genetix Ariol image analysis system, as above.

MIB-1/Ki-67-MIB-1/Ki-67 LI was assessed with the MIB-1 antibody from DAKO (Carpinteria, CA) at a 1:500 dilution, as previously described [10]. The area of highest 
labeling was identified at low power by a pathologist. 1,000 cells were counted to determine the MIB-1/Ki-67 LI.

p53 protein-Sections were deparaffinized and rehydrated in graded alcohols. Antigen retrieval was performed by heating in a pressure cooker at $100^{\circ} \mathrm{C}$ in $10 \mathrm{mM}$ sodium citrate,

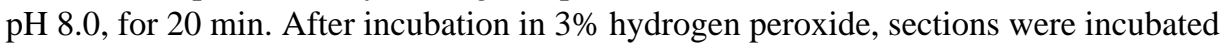
overnight at 4 degrees Celsius in primary antibody (p53, Dako (Carpinteria, CA)) at a 1:100 dilution. Staining was visualized using a kit from Dako (Carpinteria, CA).

\section{Statistical methods}

The strength of the association between the three histological markers was assessed via Spearman's correlation. The relationship between immuno-reactivity for each histological marker and time to progression (TTP) was assessed via two complementary methods. Histological results from each marker were dichotomized into two groups based around the median; survival curves were then calculated by the Kaplan-Meier method. Log rank test was used to determine if a significant difference existed between these two survival curves. A rank correlation analysis with Harrell's modification of Somer's Dxy for right-censored data was also performed to assess the strength and direction of the association between each marker and TTP. A permutation test was used to compute appropriate $p$ values. Analyses were performed with S-PLUS statistical software.

\section{Results}

\section{Study population}

From 1992 to 2006, 163 patients underwent pituitary surgery for NFPA at MDACC. One hundred and six patients met the stated criteria. Seventy-six of these eligible patients had sufficient tissue available for analysis. Among these 76 patients, 19 progression events were observed after a total of 282 person-years of follow-up. Pertinent clinical data obtained prior to initial MDACC surgery is included in Table 1.

Table 2 summarizes the 19 progression events and their pHH3 status. Six out of 9 total (67\%) patients who had progression following outside surgical resection and necessitated further surgical resection at MDACC demonstrated pHH3 immuno-reactivity. Six out of 10 total (60\%) patients who progressed after initial surgery at MDACC for de novo disease demonstrated pHH3 immuno-reactivity.

Out of the 19 patients demonstrating progression of disease, 12 underwent additional surgical resection, 1 underwent surgical resection with adjuvant radiation therapy, 4 underwent radiation therapy (an additional 1 received the recommendation to undergo radiation therapy), and 1 is currently being observed.

\section{Histological marker correlation}

A modest correlation was found between $\mathrm{p} 53$ and MIB-1/Ki-67 immuno-reactivity $(\mathrm{r}=0.25$, $p=0.031)$. P53 did not correlate with $\mathrm{pHH} 3(\mathrm{r}=0.18, p=0.12)$, nor did MIB-1/Ki-67 correlate with $\mathrm{pHH} 3(\mathrm{r}=0.11, p=0.33)$.

\section{Time to progression}

The association between each of the three biomarkers and TTP was assessed. Via log-rank analysis (Fig. 1), only MIB-1/Ki-67 above the median correlated with TTP $($ median = $0.55 \% ; p=0.018)$. P53 immuno-reactivity above the median did not correlate with a shorter TTP (median $=0.30 \% ; p=0.13$ ), nor did $\mathrm{pHH} 3$ immuno-reactivity above the median (median $=0.01 \% ; p=0.84)$. 
Rank correlation analysis with Harrell's modification demonstrated that both p53 (Dxy = $-0.40, p=0.016$ ) and MIB-1/Ki-67 (Dxy $=-0.33, p=0.036$ ) immuno-reactivity was associated with a shorter TTP. PHH3 did not appear to correlate with TTP (Dxy $=-0.04, p=$ $0.39)$.

\section{Discussion}

There is currently no commonly accepted marker to predict tumor recurrence and thus aid the clinician in assessing the need for adjuvant therapy or determining the intensity of follow-up for NFPA in the postoperative setting. The objective of this study was to assess pHH3 immuno-reactivity as a novel marker of cellular proliferation in NFPA as compared to other indices of proliferation, and to investigate the relationship between pHH3 immunoreactivity and TTP in a retrospective series of NFPA. Though previous histological markers have been explored for this explicit purpose, no marker as of yet is commonly utilized in the clinical setting.

The MIB-1/Ki-67 monoclonal antibody is immuno-reactive for the Ki-67 nuclear antigen in cells within non-G0 phases and therefore identifies cycling cells [12]. The proliferation rate of the tissue, however, depends not only on the number of cells in the cycle but also on the time it takes to complete the cycle; MIB-1/Ki-67 is limited in that it only assesses the former [13]. Interestingly, the MIB-1/Ki-67 LI is known to be affected by the presence of TSH, ACTH, prolactin, and growth hormone secretion (which introduces variability in the LI) as well as pre-operative medical treatment with dopamine agonists and somato-statin analogues (which decreases the LI) [14-16]. An inverse relationship between LI and age, and positive correlations with the presence of diabetes insipidus and visual field defects have also been observed $[8,17]$. Studies evaluating the MIB-1/Ki-67 LI and its role in clinical outcome have yielded variable results; experience has furthermore indicated that there is interlaboratory variability depending on staining protocol, tissue section thickness, and counting methods $[4,7-9,18]$. The co-expression of p53 with MIB-1/Ki-67 has additionally been explored. While p53 mutations have not been found in pituitary adenomas, p53 immunoreactivity has been found to correlate with tumor invasiveness [5]. Though p53 and MIB-1/ Ki67 immuno-histology is useful in evaluating the behavior of clinical silent pituitary adenomas, negative results do not exclude invasiveness [2].

PHH3 offers the possibility of obtaining a true mitotic index because it stains only cells in mitosis and allows the pathologist to confirm the morphologic characteristics of mitosis thereby increasing the specificity of the classification. This makes pHH3 staining less susceptible to variation based on antibody dilution or staining intensity [10, 11, 19]. Recent findings additionally make $\mathrm{pHH} 3$ an attractive anti-tumoral target. Aurora kinases have been implicated in tumourigenesis through their effects on chromosome alignment, segregation and cytokinesis during mitosis; aurora-B kinase specifically phosphorylates $\mathrm{pHH} 3$ during mitosis. Grundy et al. [20] have previously demonstrated that AZD1152-HQPA, a highly selective inhibitor of aurora-B kinase, inhibits pHH3 phosphorylation and leads to loss of viability in a panel of AML cell lines.

Our results are consistent with those from previous studies which demonstrate that both p53 and MIB-1/Ki-67 have usefulness as biomarkers in the analysis of pituitary adenomas [2, 4, $7-9,18]$. Our complementary analyses in this retrospective series of NFPA confirmed that pHH3 immuno-reactivity did not correlate with MIB-1/Ki-67 LI or p53 immuno-reactivity, and was not associated with a shorter TTP. The strength of our study includes the relatively large amount of pathological material available for analysis. Selection bias for more aggressive tumors cannot be eliminated given the nature of the referral pattern to MDACC. The relatively few number of progression events limited the robustness of the analysis. 
While our results did not confirm that pHH3 immuno-reactivity correlates with TTP, we did find it interesting that over $60 \%$ of those patients exhibiting progression of their disease demonstrated $\mathrm{pHH} 3$ immuno-reactivity. A multi-center prospective study would thus provide an ideal assessment. Our results re-confirm the utility of MIB-1/Ki-67 and p53 as markers of tumor progression in NFPA.

\section{Acknowledgments}

This study was supported by institutional (MDACC) start-up funds awarded to JKD and in part by the National Institutes of Health through the University of Texas M. D. Anderson Cancer Center's Cancer Center Support Grant CA 16672 (MEC).

\section{References}

1. Ironside JW. Pituitary gland pathology. J Clin Pathol. 2003; 56:561-568. [PubMed: 12890801]

2. Schreiber S, Saeger W, Ludecke DK. Proliferation markers in different types of clinically nonsecreting pituitary adenomas. Pituitary. 1999; 1:213-220. [PubMed: 11081200]

3. Gurlek A, Karavitaki N, Ansorge O, Wass JAH. What are the markers of aggressiveness in prolactinomas? Changes in cell biology, extracellular matrix components, angiogenesis and genetics. Eur J Endocrinol. 2007; 156:143-153. [PubMed: 17287403]

4. Honegger J, Prettin C, Feuerhake F, Petrick M, Schulte-Monting J, Reincke M. Expression of Ki-67 antigen in nonfunctioning pituitary adenomas: correlation with growth velocity and invasiveness. $\mathrm{J}$ Neurosurg. 2003; 99:674-679. [PubMed: 14567602]

5. Kontogeorgos G. Predictive markers of pituitary adenoma behavior. Neuroendocrinology. 2006; 83:179-188. [PubMed: 17047381]

6. Kontogeorgos G. Classification and pathology of pituitary tumors. Endocrine. 2005; 28:27-35. [PubMed: 16311407]

7. Hentschel SJ, McCutcheon IE, Moore W, Durity FA. p53 and MIB-1 immunohistochemistry as predictors of the clinical behavior of nonfunctioning pituitary 7adenomas. Can J Neurol Sci. 2003; 30:215-219. [PubMed: 12945944]

8. Losa M, Franzin A, Mangili F, Terreni MR, Barzaghi R, Veglia F, Mortini P, Giovanelli M. Proliferation index of nonfunctioning pituitary adenomas: correlations with clinical characteristics and long-term follow-up results. Neurosurgery. 2000; 47:1313-1319. [PubMed: 11126902]

9. Suzuki M, Minematsu T, Oyama K, Tahara S, Miyai S, Sanno N, Osamura RY, Teramoto A. Expression of proliferation markers in human pituitary incidentalomas. Endocr Pathol. 2006; 17:263-276. [PubMed: 17308363]

10. Colman H, Giannini C, Huang L, Gonzalez J, Hess K, Bruner J, Fuller G, Langford L, Pelloski C, Aaron J, Burger P, Aldape K. Assessment and prognostic significance of mitotic index using the mitosis marker phoso-histone $\mathrm{H} 3$ in low and intermediate-grade infiltrating astrocytomas. Am J Surg Pathol. 2006; 30:657-664. [PubMed: 16699322]

11. Ribalta T, McCutcheon IE, Aldape KD, Bruner JM, Fuller GN. The mitosis-specific antibody antiphosphohistone-H3 (PHH3) facilitates rapid reliable grading of meningiomas according to WHO 2000 criteria. Am J Surg Pathol. 2004; 28:1532-1536. [PubMed: 15489659]

12. Prevedello DM, Jagannathan J, John JA Jr, Lopes BS, Laws ER Jr. Relevance of high Ki-67 in pituitary adenomas. Neurosurg Focus. 2005; 19:E11. [PubMed: 16398461]

13. Turner HE, Wass JAH. Are markers of proliferation valuable in the histological assessment of pituitary tumors? Pituitary. 1999; 1:147-151. [PubMed: 11081192]

14. Jaffrain-Rea ML, Di Stefano D, Minniti G, Esposita V, Bultrini A, Ferretti E, Santoro A, Faticanti Scucchi L, Gulino A, Cantore G. A critical reappraisal of MIB-1 labelling index significance in a large series of pituitary tymours: secreting versus non-secreting adenomas. Endocr Relat Cancer. 2002; 9:103-113. [PubMed: 12121834]

15. Scheithauer BW, Gaffey TA, Lloyd V, Sebo TJ, Kovacs KT, Horvath E, Yapicier O, Young WF Jr, Meyer FB, Kuroki T, Riehle DL, Laws ER Jr. Pathobiology of pituitary adenomas and carcinomas. Neurosurgery. 2006; 59:341-353. [PubMed: 16883174] 
16. Wolfsberger S, Wunderer J, Zachenhofer I, Czech T, Bocher-Schwarz HG, Hainfellner J, Knosp E. Expression of cell proliferation markers in pituitary adenomas-correlation and clinical relevance of MIB-1 and anti-topoisomerase-IIalpha. Acta Neurochir. 2004; 146:831-839. [PubMed: 15254805]

17. Paek KI, Kim SH, Song SH, Choi SW, Koh HS, Youm JY, Kim Y. Clinical significance of Ki-67 labeling index in pituitary macroadenomas. J Korean Med Sci. 2005; 20:489-494. [PubMed: 15953875]

18. Fillipella M, Galland F, Kujas M, Young J, Faggiano A, Lombardi G, Colao A, Meduri G, Chanson P. Pituitary tumor transforming gene (PTTG) expression correlates with the proliferative activity and recurrence status of pituitary adenomas: a clinical and immunohistochemical study. Clin Endocrinol. 2006; 65:536-543.

19. Takei H, Battacharjee MB, Rivera A, Dancer Y, Powerll SZ. New immunohistochemical markers in the evaluation of central nervous system tumors. Arch Pathol Lab Med. 2007; 131:234-241. [PubMed: 17284108]

20. Grundy M, Seedhouse C, Shang S, Richardson J, Russell N, Pallis M. The FLT3 internal tandem duplication mutation is a secondary target of the aurora B kinase inhibitor AZD1152-HQPA in acute myelogenous leukemia cells. Mol Cancer Ther. 2010; 9:661-672. [PubMed: 20159992] 

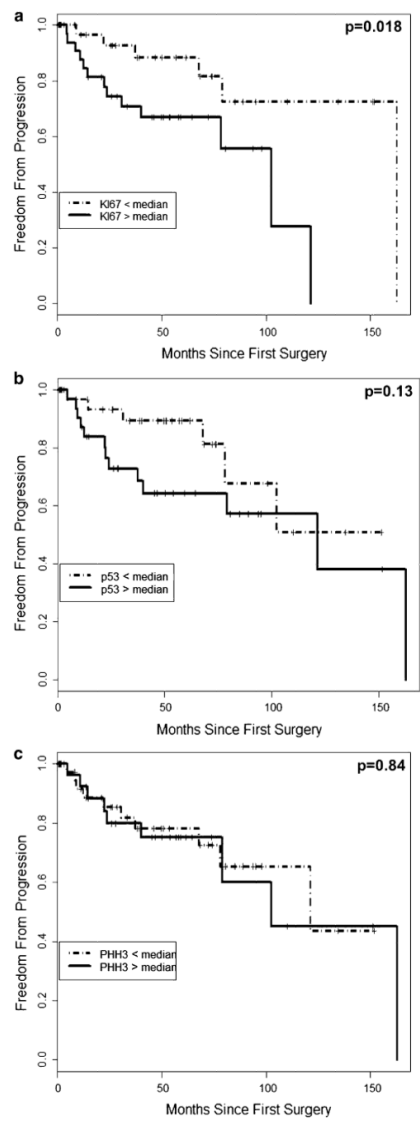

Fig. 1.

Kaplan-Meier curves and log-rank test $p$ values for each marker dichotomized at the median. a MIB-1/Ki-67 (median 0.55\%), b p53 (median 0.30\%), c PHH3 (median 0.01\%) 
Table 1

Clinical characteristics of the 76 patients included in the series

\begin{tabular}{ll}
\hline Patient population & $\mathbf{N}(\boldsymbol{\%})$ \\
\hline Total number of patients included & 76 \\
Median age at first surgery, years (range) & $50.9(24-78)$ \\
Female & $29(38)$ \\
Male & $47(62)$ \\
Initial tumor size & \\
$<1$ cm & $2(3)$ \\
$\geq 1$ cm & $74(97)$ \\
Invasiveness & \\
Intrasellar only & $9(12)$ \\
Intrasellar with: & \\
$\quad$ Cavernous sinus extension & $0(0)$ \\
$\quad$ Suprasellar extension & $44(58)$ \\
$\quad$ Both cavernous sinus and suprasellar extension & $17(22)$ \\
$\quad$ Bony erosion & $2(3)$ \\
$\quad$ Suprasellar extension and bony erosion & $1(1)$ \\
$\quad$ Suprasellar extension, cavernous sinus extension, and bony erosion & $3(4)$ \\
Clinicopathologic diagnosis & \\
Pituitary adenoma & $52(69)$ \\
Silent somatotroph & $1(1)$ \\
Silent lactotroph & $3(4)$ \\
Silent thyrotroph & $1(1)$ \\
Silent corticotroph & $1(1)$ \\
Silent gonadotroph & $18(24)$ \\
Vre-operative visual field defects & \\
No visual field defects present & $29(38)$ \\
\hline & $47(62)$ \\
\hline
\end{tabular}


Table 2

PHH3 immuno-reactivity in the 19 patients with progressive disease (PD)

\begin{tabular}{lll}
\hline & PHH3 positive (N) & PHH3 negative (N) \\
\hline Patients with PD after initial MDACC surgery & 6 & 4 \\
Patients with PD after surgery at outside institution—necessitating further surgery at MDACC & 6 & 3 \\
\hline
\end{tabular}

